\title{
On Linear Processing for Dual-hop Multi-channel Relaying
}

\author{
Min Dong*, Mahdi Hajiaghayi ${ }^{\dagger}$, and Ben Liang ${ }^{\dagger}$ \\ ${ }^{*}$ Faculty of Engineering and Applied Science, University of Ontario Institute of Technology, Canada \\ ${ }^{\dagger}$ Dept. of Electrical and Computer Engineering, University of Toronto, Canada \\ Email: min.dong@uoit.ca, \{mahdih, liang\}@comm.utoronto.ca
}

\begin{abstract}
In this paper, we consider the amplified-and-forward relaying in a multichannel system with linear processing capability at the relay. We propose an analytical approach to study the linear processing performance with an aim to maximize the end-to-end achievable rate, assuming equal power amplification at the relay. For the class of permutation matrices as the special case of linear processing, the problem reduces to finding the optimal channel pairing scheme that maps incoming channels to outgoing channels at the relay. The proposed unified approach allows us to obtain the corresponding optimal permutation for channel pairing, for either relaying with or without the direct path available. Particular to the case when the direct path is available, such optimal pairing strategy has not been shown before. We further demonstrate that the so obtained optimal permutation is in fact also optimal among all unitary matrices for achievable rate maximization, thus, establishing the optimality of channel pairing approach among unitary linear processing schemes. Simulation results are presented to demonstrate the achievable gain of optimal channel pairing compared with nonoptimal linear processing and non-pairing.
\end{abstract}

\section{INTRODUCTION}

Multichannel-based relaying combines the advantages of both broadband multichannel communication and relaying techniques to improve network performance and efficiency. It is essential that the relaying techniques can be maximally explored in such system. In a narrow-band single channel system, the relay retransmits the processed version of the received signal over the same channel. For a multicahnnel system such as OFDM, the relay has an additional frequency dimension to exploit and process the incoming signals adaptively based on the strength of each channels for relaying purpose. Such exploitation can potentially improve the overall relay performance. To maintain low-complexity processing at the relay, we focus on linear processing of incoming signals. In this paper, we address how to optimally perform channel aware linear processing of the incoming signals at the relay to maximize the relaying performance in a multichannel system.

Channel pairing, which maps incoming and outgoing channels at the relay, along with power allocation, can be viewed as a special case of linear processing. It was first proposed independently in [1] and [2] for an amplified-and-forward (AF) dual-hop OFDM relay system. For relaying without the direct source-destination path available, [1] used integer programming to find the optimal pairing that maximizes the sum SNR, whereas from a system-design perspective, [2] proposed a sorted SNR pairing scheme optimal in the noise- free case, assuming uniform power allocation. Subsequently, the sorted SNR pairing scheme is shown optimal in the noisy relaying case [3]. Furthermore, channel pairing was studied in MIMO OFDM systems [4], as well as in decode-andforward (DF) relay systems [5]. Joint channel pairing and power allocation for single-user relaying was studied in [6] and [7] for dual-hop DF relaying in an OFDM system. For multi-hop relaying, jointly optimal channel pairing and power allocation were established in [8]. For relaying with direct path available, joint optimization of channel pairing and power allocation were studied in [9] for single-user relaying, and the jointly optimal channel-user assignment, channel pairing, and power allocation scheme was proposed in [10] for multiuser relaying. In both cases, algorithms are designed to find a jointly optimal solution, although no explicit channel pairing strategy can be found. Except [9], [10], all of the above works focus on the relay path only, without direct-path transmission, perhaps partially due to the difficulty in finding optimal channel pairing in the case with direct path. Channel pairing aside, there is little study on the general linear processing of incoming signals at the relay and its impact on the end-toend performance. In particular, with much attention on finding the optimal channel pairing scheme for relaying, the natural questions arise on how good the performance of channel pairing is compared to other linear processing schemes, and whether there exist conditions such that pairing is optimal. Until now, there is very little understanding on these issues and much is unknown.

In this paper, we consider the problem of linear processing at the relay for a multichannel relaying system. A half-duplex dual-hop AF relay is considered, where the incoming signals across channels at the relay are processed with a linear scheme, and the relay then forwards the amplified version of the processed signals. We propose an analytical approach to study the linear processing performance that maximizes the endto-end achievable rate, assuming equal power amplification at the relay. For the class of permutation matrices as the special case of linear processing, the problem reduces to finding the optimal channel pairing scheme at the relay. The proposed unified approach allows us to obtain the explicit optimal channel pairing strategy for relaying with the direct path available, which was not known before. We further demonstrate that, among all unitary matrices, the so obtained optimal permutation for channel pairing is in fact optimal for 
achievable rate maximization, thus, establishing the optimality of channel pairing approach among unitary linear processing schemes.

The rest of this paper is organized as follows. In Section II, we present the system model and problem formulation. Section III presents our analytical framework to determine the optimal permutation matrix and hence the optimal channel pairing strategy. In Section IV, we establish the optimality of channel pairing among unitary linear processing schemes. We present simulation results to demonstrate the performance gain achieved through optimal channel pairing in Section $\mathrm{V}$ and finally conclude in Section VI.

\section{Problem Statement}

\section{A. System Model}

We consider a dual-hop relay network with a pair of source and destination nodes and a single AF relay node in a multichannel system with $N$ channels. We constrain ourselves to half-duplex transmission, where a relay node is either in transmission or reception but not simultaneously. The cooperative transmission takes place in two phases. In the first phase the source sends data through $N$ channels to the relay and destination simultaneously (if the direct path is available). The relay then performs linear processing of the received signals over these $N$ incoming channels, and forwards the amplified version of the processed signals to the destination.

We denote the channel gain over channel $k$ from source to destination, from source to relay, and from relay to destination by $h_{0 k}, h_{1 k}$, and $h_{2 k}$, respectively, and $s_{k}$ the source signal transmitted on channel $k$ with power coefficient $d_{s k}$. The received signals at the relay and destination in the first phase are given by

$$
\begin{aligned}
\mathbf{y}_{r} & =\mathbf{H}_{1} \mathbf{D}_{s} \mathbf{s}+\mathbf{n}_{r} \\
\mathbf{y}_{d}^{(1)} & =\mathbf{H}_{0} \mathbf{D}_{s} \mathbf{s}+\mathbf{n}_{d}^{(1)}
\end{aligned}
$$

where $\mathbf{y}_{r}=\left[y_{r 1}, \cdots, y_{r N}\right]^{T}$ and $\mathbf{y}_{d}^{(1)}=\left[y_{d 1}^{(1)}, \cdots, y_{d N}^{(1)}\right]^{T}$ are the received signal vector at the relay and destination, respectively, $\mathbf{H}_{1}=\operatorname{diag}\left(h_{11}, \cdots, h_{1 N}\right)$ and $\mathbf{H}_{0}=$ $\operatorname{diag}\left(h_{01}, \cdots, h_{0 N}\right)$ are the corresponding channel matrices, and $\mathbf{D}_{s}=\operatorname{diag}\left(\mathbf{d}_{s}\right)$ with $\mathbf{d}_{s}=\left[d_{s 1}, \cdots, d_{s N}\right]^{T}$ being the vector of transmit power coefficients. The signals in $\mathbf{s}$ are i.i.d. with average unit power: $\mathrm{E}\left[\mathbf{s s}^{H}\right]=\mathbf{I}$. Moreover, $\mathbf{n}_{r}=\left[n_{r 1}, \cdots, n_{r N}\right]^{T}$ and $\mathbf{n}_{d}^{(1)}=\left[n_{d 1}^{(1)}, \cdots, n_{d N}^{(1)}\right]^{T}$ are AWGN at the relay and the destination, with $\mathbf{n}_{r} \sim \mathcal{C N}\left(0, \sigma_{r}^{2} \mathbf{I}\right)$ and $\mathbf{n}_{d}^{(1)} \sim \mathcal{C N}\left(0, \sigma_{d}^{2} \mathbf{I}\right)$, respectively.

In the second phase, the received signal $\mathbf{y}_{r}$ is first linearly combined, and then the relay retransmits the amplified version of the processed signal to the destination. Here, in order to separate the processing effects, we break the process into two steps, the (normalized) linear combining and power amplification. Thus, the retransmitted signal is given by $\mathbf{D}_{r} \mathbf{W} \mathbf{y}_{r}$, where $\mathbf{W}$ is the linear processing matrix, and $\mathbf{D}_{r}=\operatorname{diag}\left(\mathbf{d}_{r}\right)$ is the power amplification matrix with $\mathbf{d}_{r}$ being the power amplification vector for the processed signal at the relay. The received signal vector at the destination is given by

$$
\begin{aligned}
\mathbf{y}_{d}^{(2)} & =\mathbf{H}_{2} \mathbf{D}_{r} \mathbf{W} \mathbf{y}_{r}+\mathbf{n}_{d}^{(2)} \\
& =\mathbf{H}_{2} \mathbf{D}_{r} \mathbf{W}\left(\mathbf{H}_{1} \mathbf{D}_{s} \mathbf{s}+\mathbf{n}_{r}\right)+\mathbf{n}_{d}^{(2)}
\end{aligned}
$$

where $\mathbf{y}_{d}^{(2)}=\left[y_{d 1}^{(2)}, \cdots, y_{d N}^{(2)}\right]^{T}, \mathbf{H}_{2}=\operatorname{diag}\left(h_{21}, \cdots, h_{2 N}\right)^{T}$, and $\mathbf{n}_{d}^{(2)}=\left[n_{d 1}^{(2)}, \cdots, n_{d N}^{(2)}\right]^{T} \sim \mathcal{C N}\left(0, \sigma_{d}^{2} \mathbf{I}\right)$.

In this paper, we consider the class of unitary processing matrices for $\mathbf{W}$. We also assume that the relay equally amplifies the processed signal over each channel, i.e., $\mathbf{D}_{r}$ is a scalar of identity matrix $\mathbf{D}_{r}=d_{r} \mathbf{I}$. Let $P_{r}$ be the average power budget at the relay. The processing matrix $\mathbf{W}$ and the power amplification matrix $\mathbf{D}_{r}$ at the relay must satisfy

$$
\mathrm{E}\left\|\mathbf{D}_{r} \mathbf{W}\left(\mathbf{H}_{1} \mathbf{D}_{s} \mathbf{s}\right)\right\|^{2}+\mathrm{E}\left\|\mathbf{D}_{r} \mathbf{W} \mathbf{n}_{r}\right\|^{2} \leq P_{r}
$$

which, under our assumptions, leads to

$$
d_{r}=\sqrt{\frac{P_{r}}{\sum_{k=1}^{N} d_{s k}^{2}\left|h_{1 k}\right|^{2}+N \sigma_{r}^{2}}} .
$$

Note that, since $\mathbf{W}$ is unitary, it does not appear in (4).

Let $P_{s}$ be the power budget at the source. Then, the transmit power matrix $\mathbf{D}_{s}$ must satisfy

$$
\mathrm{E}\left\|\mathbf{D}_{s} \mathbf{s}\right\|^{2}=\left\|\mathbf{d}_{s}\right\|^{2} \leq P_{s} .
$$

In this study, we focus on the effect of the processing matrix $\mathbf{W}$ on the relay performance, and assume a pre-determined power allocation at the source, i.e., $\mathbf{D}_{s}$ is given.

\section{B. Linear Processing and Achievable Rate}

We consider the achievable rate obtained through the above described AF relaying with linear processing in the multichannel system. Our goal is to find the optimal $\mathbf{W}^{*}$ to maximize such achievable rate. Regardless of whether the direct path is available or not, we can rewrite the end-to-end system equation in the following general form

$$
\mathbf{y}=\widetilde{\mathbf{H}}(\mathbf{W}) \mathbf{s}+\widetilde{\mathbf{n}}(\mathbf{W})
$$

where $\widetilde{\mathbf{H}}(\mathbf{W})$ and $\widetilde{\mathbf{n}}(\mathbf{W})$ are the equivalent channel matrix and the equivalent noise term, respectively. They are functions of the processing matrix $\mathbf{W}$. Given the system described earlier, the system achievable rate is given by [11]

$$
R(\mathbf{W})=\frac{1}{2} \log \operatorname{det}\left(\mathbf{I}+\mathbf{R}_{n}^{-1} \widetilde{\mathbf{H}}(\mathbf{W}) \widetilde{\mathbf{H}}^{H}(\mathbf{W})\right),
$$

where $\mathbf{R}_{n}=\mathrm{E}\left[\widetilde{\mathbf{n}}(\mathbf{W}) \widetilde{\mathbf{n}}^{H}(\mathbf{W})\right]$ is the covariance matrix of the equivalent noise term. The factor $1 / 2$ reflects the half-duplex operation.

Our goal is to find the optimal $\mathbf{W}^{*}$ to maximize the achievable rate

$$
\mathbf{W}^{*}=\underset{\mathbf{W}: \mathbf{W} \mathbf{W}^{H}=\mathbf{W}^{H} \mathbf{W}=\mathbf{I}}{\arg \max } \log \operatorname{det}\left(\mathbf{I}+\mathbf{R}_{n}^{-1} \widetilde{\mathbf{H}}(\mathbf{W}) \widetilde{\mathbf{H}}^{H}(\mathbf{W})\right) .
$$

For the conventional multichannel relaying without linear processing, i.e., $\mathbf{W}=\mathbf{I}$, the relay simply forwards the amplified signal to the destination over the same channel. 
However, such forwarding is in general not optimal in terms of maximizing the achievable rate (under the $\mathrm{AF}$ relaying protocol). As mentioned earlier, a special class of $\mathbf{W}$ is the permutation matrix $\Pi$, for which linear processing reduces to channel pairing. Such a scheme would uniquely couple a channel over the first hop with a possibly different channel over the second hop for signal relaying. This technique was studied recently in a few specific relay models [1], [2], [12] and was shown to improve the end-to-end data rate. However, whether such approach is optimal among all possible linear combining, and under what condition it is optimal, remain unclear.

In the following, we will first focus on the class of permutation matrices $\{\boldsymbol{\Pi}\}$ to find the optimal permutation, for relaying with and without direct path. We will then discuss the optimal $\mathbf{W}^{*}$ for these scenarios.

\section{Linear Processing through PeRmutation: ChANNEl PAIRING}

To solve (7), we first focus on the class of permutation matrices $\{\mathbf{W}=\Pi\}$, and propose an approach to find the optimal $\Pi^{*}$ for channel pairing. This approach relies on the following result

Lemma 1: Let $\mathbf{P}$ and $\mathbf{Q}$ be two diagonal matrices. For

$$
\max _{\boldsymbol{\Pi}} \operatorname{det}\left(\mathbf{I}+(\mathbf{P} \boldsymbol{\Pi} \mathbf{Q})^{H}(\mathbf{P} \boldsymbol{\Pi} \mathbf{Q})\right)
$$

among all possible permutation matrices $\Pi$, the optimal $\Pi^{*}$ is the one that maps the sorted absolute values of the diagonal entries of $\mathbf{P}$ to the sorted absolute value of the diagonal entries of $\mathbf{Q}$.

To show the above, we see that the objective function in (8) can be rewritten as $\prod_{i=1}^{n}\left(1+\left|p_{i}\right|^{2}\left|q_{(i)_{\Pi}}\right|^{2}\right)$, where $p_{i}$ and $q_{i}$ are the diagonal elements of $\mathbf{P}$ and $\mathbf{Q}$, respectively. Function $(i)_{\Pi}$ maps the index $i$ after permutation to the original index. Let $\left\{\left|p_{(i)}\right|^{2}\right\}$ and $\left\{\left|q_{(i)}\right|^{2}\right\}$ be the corresponding ordered sequences. Then it is not difficult to show that $\prod_{i=1}^{n}\left(1+\left|p_{i}\right|^{2}\left|q_{(i)_{\Pi}}\right|^{2}\right) \leq$ $\prod_{i=1}^{n}\left(1+\left|p_{(i)}\right|^{2}\left|q_{(i)}\right|^{2}\right)$.

\section{A. Optimal Pairing for Relay without Direct Path}

In this case, the destination is out of the transmission zone of the source. From (3), the equivalent channel matrix, noise vector, and its covariance matrix in (5) are given by

$$
\begin{aligned}
\widetilde{\mathbf{H}}(\boldsymbol{\Pi}) & =\mathbf{H}_{2} \mathbf{D}_{r} \boldsymbol{\Pi} \mathbf{H}_{1} \mathbf{D}_{s}, \\
\widetilde{\mathbf{n}}(\boldsymbol{\Pi}) & =\mathbf{H}_{2} \mathbf{D}_{r} \boldsymbol{\Pi} \mathbf{n}_{r}+\mathbf{n}_{d}^{(2)} \\
\mathbf{R}_{n} & =\sigma_{r}^{2} \mathbf{D}_{r}^{2} \mathbf{H}_{2} \mathbf{H}_{2}^{H}+\sigma_{d}^{2} \mathbf{I} .
\end{aligned}
$$

Note that, $\mathbf{R}_{n}$ is not a function of $\boldsymbol{\Pi}$. Note also that, except $\boldsymbol{\Pi}$, all matrices in (9) are diagonal. Using the property of the determinant, $\operatorname{det}(\mathbf{I}+\mathbf{A B})=\operatorname{det}(\mathbf{I}+\mathbf{B A})$, from (6), we can write the end-to-end achievable rate as

$$
R(\boldsymbol{\Pi})=\frac{1}{2} \log \operatorname{det}\left(\mathbf{I}+\widetilde{\mathbf{H}}(\boldsymbol{\Pi})^{H} \mathbf{R}_{n}^{-1} \widetilde{\mathbf{H}}(\boldsymbol{\Pi})\right)
$$

Inserting the expression of $\mathbf{R}_{n}$ in (10), we have

$$
\begin{aligned}
& R(\boldsymbol{\Pi})=\frac{1}{2} \times \\
& \log \operatorname{det}(\mathbf{I}+(\underbrace{\mathbf{R}_{n}^{-\frac{1}{2}} \mathbf{H}_{2} \mathbf{D}_{r}}_{\mathbf{P}} \boldsymbol{\Pi} \underbrace{\mathbf{H}_{1} \mathbf{D}_{s}}_{\mathbf{Q}})^{H} \underbrace{\mathbf{R}_{n}^{-\frac{1}{2}} \mathbf{H}_{2} \mathbf{D}_{r}}_{\mathbf{P}} \boldsymbol{\Pi} \underbrace{\mathbf{H}_{1} \mathbf{D}_{s}}_{\mathbf{Q}}) .
\end{aligned}
$$

The $i$-th diagonal entries $p_{i}$ and $q_{i}$ of $\mathbf{P}$ and $\mathbf{Q}$ are $p_{i}=$ $\frac{h_{2 i} d_{r}}{\sqrt{\sigma_{d}^{2}+\sigma_{r}^{2}\left|h_{2 i} d_{r}\right|^{2}}}$ and $q_{i}=h_{1 i} d_{s i}$, respectively. Following Lemma 1 , the optimal $\Pi^{*}$ is to pair the ordered sequences of $\left\{\left|p_{i}\right|\right\}$ and $\left\{\left|q_{i}\right|\right\}$, or equivalently, to pairing the following two ordered sequences

$$
\left\{\frac{\mathrm{SNR}_{r d, i}}{1+\sigma_{r}^{2} \mathrm{SNR}_{r d, i}}\right\}, \quad\left\{\mathrm{SNR}_{s r, i}\right\}
$$

where $\mathrm{SNR}_{s r, i}=\frac{\left|h_{1 i}\right|^{2} d_{s i}^{2}}{\sigma_{r}^{2}}$ and $\mathrm{SNR}_{r d, i}=\frac{\left|h_{2 i}\right|^{2} d_{r}^{2}}{\sigma_{d}^{2}}$ are the received SNR from source to relay, and from relay to destination, over the $i$ th subcarrier, respectively.

Note that, because $f(x)=\frac{x}{\sqrt{a x^{2}+b}}$ is monotonically increasing for $a>0$, ordering $\left\{\left|p_{i}\right|\right\}$ is equivalent to ordering $\left\{\left|h_{2 i} d_{r}\right|\right\}$, or equivalently $\mathrm{SNR}_{r d, i}$. In other words, the optimal pairing in this case reduces to pairing based on sorted SNR. This optimal pairing has been shown under both noise-free relaying $\left(\sigma_{r}^{2}=0\right)$ [2] and noisy relaying [3] in OFDM systems, under given power allocation. We show that the same optimality can be shown with our approach in this setup. Furthermore, as we will see in the following, our approach allows us to find the optimal pairing when direct path is available. This is the case where the optimal pairing was not known.

\section{B. Optimal Pairing for Relay with Direct Path}

We now consider the case when the direct path is available. The received signals at the destination can be written as $\mathbf{y}=$ $\left[\mathbf{y}_{d}^{(1) H} \mathbf{y}_{d}^{(2) H}\right]^{H}$. In this case, we have

$$
\begin{aligned}
\tilde{\mathbf{H}}(\boldsymbol{\Pi}) & =\left[\begin{array}{c}
\mathbf{H}_{o} \\
\mathbf{H}_{2} \mathbf{D}_{r} \boldsymbol{\Pi} \mathbf{H}_{1} \mathbf{D}_{s}
\end{array}\right], \\
\tilde{\mathbf{n}}(\boldsymbol{\Pi}) & =\left[\begin{array}{c}
\mathbf{n}_{d}^{(1)} \\
\mathbf{H}_{2} \mathbf{D}_{r} \boldsymbol{\Pi} \mathbf{n}_{r}+\mathbf{n}_{d}^{(2)}
\end{array}\right] .
\end{aligned}
$$

The achievable rate in (6) for this case is given by ${ }^{1}$

$$
R(\boldsymbol{\Pi})=\frac{1}{2} \log \operatorname{det}\left(\mathbf{I}+\left(\boldsymbol{\Upsilon}_{2} \boldsymbol{\Pi} \boldsymbol{\Upsilon}_{1}\right)^{H} \boldsymbol{\Upsilon}_{2} \boldsymbol{\Pi} \boldsymbol{\Upsilon}_{1}+\boldsymbol{\Upsilon}_{0}^{H} \boldsymbol{\Upsilon}_{0}\right) \text {, }
$$

where

$$
\begin{aligned}
& \boldsymbol{\Upsilon}_{0} \triangleq \frac{1}{\sigma_{d}} \mathbf{H}_{0} \mathbf{D}_{s}, \\
& \boldsymbol{\Upsilon}_{\mathbf{1}} \triangleq \mathbf{H}_{1} \mathbf{D}_{s}, \\
& \boldsymbol{\Upsilon}_{\mathbf{2}} \triangleq \mathbf{R}_{n}^{-\frac{1}{2}} \mathbf{H}_{2} \mathbf{D}_{r},
\end{aligned}
$$

${ }^{1}$ When a permutation matrix is used at the relay, the rate in (14) can be achieved by maximum ratio combining of the signals from the relay and the direct path. 
and $\mathbf{R}_{n}$ is given in (9). To find the optimum $\boldsymbol{\Pi}^{*}$ to maximize $R(\boldsymbol{\Pi})$, we again apply the result in Lemma 1 , and all we need is to find the equivalent $\mathbf{P}$ and $\mathbf{Q}$ to express (14) as the form in (8). To do so, we re-arrange (14) as

$$
\begin{aligned}
& R(\mathbf{\Pi})=\frac{1}{2} \log \operatorname{det}\left[\left(\mathbf{I}+\boldsymbol{\Upsilon}_{0}^{H} \boldsymbol{\Upsilon}_{0}\right) \times\right. \\
& \left.\left(\mathbf{I}+\left(\mathbf{I}+\boldsymbol{\Upsilon}_{0}^{H} \boldsymbol{\Upsilon}_{0}\right)^{-1}\left(\boldsymbol{\Upsilon}_{2} \boldsymbol{\Pi} \boldsymbol{\Upsilon}_{1}\right)^{H} \boldsymbol{\Upsilon}_{2} \boldsymbol{\Pi} \boldsymbol{\Upsilon}_{1}\right)\right] \\
& =\frac{1}{2}\left[\log \operatorname{det}\left(\mathbf{I}+\Upsilon_{0}^{H} \Upsilon_{0}\right)+\right. \\
& \left.\log \operatorname{det}\left(\mathbf{I}+\left(\boldsymbol{\Upsilon}_{2} \boldsymbol{\Pi} \boldsymbol{\Upsilon}_{1}\right)\left(\mathbf{I}+\boldsymbol{\Upsilon}_{0}^{H} \boldsymbol{\Upsilon}_{0}\right)^{-1}\left(\boldsymbol{\Upsilon}_{2} \boldsymbol{\Pi} \boldsymbol{\Upsilon}_{1}\right)^{H}\right)\right] \text {, }
\end{aligned}
$$

where the second term of (16) follows from the property $\operatorname{det}(\mathbf{I}+\mathbf{A B})=\operatorname{det}(\mathbf{I}+\mathbf{B A})$. Since the first term of (16) is independent of $\boldsymbol{\Pi}$, we are only interested in the second term as a function of $\Pi$, which can be written as

$$
\begin{aligned}
R_{2}(\boldsymbol{\Pi}) \triangleq & \frac{1}{2} \log \operatorname{det}\left(\mathbf{I}+\boldsymbol{\Upsilon}_{2} \boldsymbol{\Pi} \boldsymbol{\Upsilon}_{1}\left(\mathbf{I}+\boldsymbol{\Upsilon}_{0}^{H} \boldsymbol{\Upsilon}_{0}\right)^{-\frac{1}{2}}\right. \\
& \left.\left(\boldsymbol{\Upsilon}_{2} \boldsymbol{\Pi} \boldsymbol{\Upsilon}_{1}\left(\mathbf{I}+\boldsymbol{\Upsilon}_{0}^{H} \boldsymbol{\Upsilon}_{0}\right)^{-\frac{1}{2}}\right)^{H}\right)
\end{aligned}
$$

Again using $\operatorname{det}(\mathbf{I}+\mathbf{A B})=\operatorname{det}(\mathbf{I}+\mathbf{B A})$, and noticing that $\boldsymbol{\Upsilon}_{0}, \boldsymbol{\Upsilon}_{1}$, and $\boldsymbol{\Upsilon}_{2}$ are all diagonal matrices, we can set $\mathbf{P}=\boldsymbol{\Upsilon}_{2}$ and $\mathbf{Q}=\boldsymbol{\Upsilon}_{1}\left(\mathbf{I}+\boldsymbol{\Upsilon}_{0}^{H} \boldsymbol{\Upsilon}_{0}\right)^{-\frac{1}{2}}$, and (17) can then be transformed into the form of (8). Based on this, we obtain the optimal channel pairing scheme for relaying with direct path available.

Examining the diagonal entries of $\mathbf{P}$ and $\mathbf{Q}$, we conclude that the optimal pairing is essentially to order the following quantities over the incoming and outgoing channels at the relay, respectively,

$$
\left\{\frac{\mathrm{SNR}_{s r, i}}{1+\mathrm{SNR}_{s d, i}}\right\}, \quad\left\{\frac{\mathrm{SNR}_{r d, i}}{1+\sigma_{r}^{2} \mathrm{SNR}_{r d, i}}\right\}
$$

where $\mathrm{SNR}_{s r, i}=\frac{\left|h_{1 i}\right|^{2} d_{s i}^{2}}{\sigma_{r}^{2}}, \mathrm{SNR}_{r d, i}=\frac{\left|h_{2 i}\right|^{2} d_{r}^{2}}{\sigma_{d}^{2}}$, and $\mathrm{SNR}_{s d, i}=\frac{\left|h_{0 i}\right|^{2} d_{s i}^{2}}{\sigma_{d}^{2}}$, are the received SNR from source to relay, from relay to destination, and from source to destination, over the $i$ th channel, respectively. Again, sorting $\left\{\frac{\mathrm{SNR}_{r d, i}}{1+\sigma_{r}^{2} \mathrm{SNR}_{r d, i}}\right\}$ is equivalent to sorting $\left\{\mathrm{SNR}_{r d, i}\right\}$. Thus, when direct path is available, the optimal pairing has a very clear strategy based on the SNR on each path: it is to match incoming and outgoing channels at the relay, according to the ordering of SNR strength on the relay-destination channels, and the relative ratio of SNR strengths on the source-relay and source-destination paths. Various sorting algorithms can be employed with the computational complexity of $\mathcal{O}(N \log N)$ [13].

\section{THE OPTIMAL $\mathbf{W}^{*}$}

In the previous section, we have considered the special class of permutation matricis where $\mathbf{W}=\boldsymbol{\Pi}$, and we have obtained the optimal $\Pi^{*}$ in different relay scenarios using the proposed framework. Back to the general problem in (7), the following result shows that the optimal permutation $\Pi^{*}$ is in fact the optimal $\mathbf{W}^{*}$ among unitary matrices.
Proposition 1: Let $\mathbf{P}$ and $\mathbf{Q}$ be two diagonal matrices. The solution to the following maximization

$$
\max _{\mathbf{W}: \mathbf{W} \mathbf{W}^{H}=\mathbf{W}^{H} \mathbf{W}=\mathbf{I}} \operatorname{det}\left(\mathbf{I}+(\mathbf{P} \mathbf{W} \mathbf{Q})^{H}(\mathbf{P} \mathbf{W} \mathbf{Q})\right)
$$

is $\mathbf{W}^{*}=\boldsymbol{\Pi}^{*}$, where $\boldsymbol{\Pi}^{*}$ is the solution in Lemma 1 .

Proof: We prove this by induction. We provide a brief description of the steps leading to the conclusion.

For $N=1$, the result is trivial to show. Assume this is true for $N=n-1$. For $N=n$, Let $\mathbf{A}_{n}=$ $\left(\mathbf{P}_{n} \mathbf{W}_{n} \mathbf{Q}_{n}\right)^{H}\left(\mathbf{P}_{n} \mathbf{W}_{n} \mathbf{Q}_{n}\right)$, where subscript $n$ denotes the matrix dimension. Since $\left(\mathbf{I}_{n}+\mathbf{A}_{n}\right)$ is a positive definite matrix, it has the following property [14]

$$
\operatorname{det}\left(\mathbf{I}_{n}+\mathbf{A}_{n}\right) \leq\left(1+a_{n n}\right) \operatorname{det}\left(\mathbf{I}_{n-1}+\mathbf{A}_{n-1}\right),
$$

where $a_{n n}=\left(\mathbf{A}_{n}\right)_{n n}$. Re-arranging the expression of the determinant, we have

$$
\operatorname{det}\left(\mathbf{I}_{n}+\mathbf{A}_{n}\right)=\operatorname{det}\left(\mathbf{I}_{n}+\mathbf{W}^{H} \mathbf{Q}^{H} \mathbf{Q} \mathbf{W} \mathbf{P} \mathbf{P}^{H}\right) .
$$

Let $\mathbf{W}=\left(\begin{array}{lll}\mathbf{w}_{1} & \cdots & \mathbf{w}_{n}\end{array}\right), \mathbf{P}=\operatorname{diag}(\mathbf{p})$ with $\mathbf{p}=$ $\left[p_{1}, \cdots, p_{n}\right]^{T}$, and $\mathbf{Q}=\operatorname{diag}\left(q_{1}, \cdots, q_{n}\right)$. Let $\odot$ denote the Hadamard product. We have

$$
\begin{gathered}
\mathbf{A}_{n}=\left(\begin{array}{ccc}
\left(|\mathbf{p}|^{2} \odot \mathbf{w}_{1}\right)^{H}\left|q_{1}\right|^{2} \mathbf{w}_{1} & \cdots & \left(|\mathbf{p}|^{2} \odot \mathbf{w}_{1}\right)^{H}\left|q_{n}\right|^{2} \mathbf{w}_{n} \\
\vdots & \ddots & \vdots \\
\left(|\mathbf{p}|^{2} \odot \mathbf{w}_{n}\right)^{H}\left|q_{1}\right|^{2} \mathbf{w}_{1} & \cdots & \left(|\mathbf{p}|^{2} \odot \mathbf{w}_{n}\right)^{H}\left|q_{n}\right|^{2} \mathbf{w}_{n}
\end{array}\right) \\
=\left(\begin{array}{cc}
\mathbf{A}_{n-1} & |\mathbf{p}|^{2} \odot\left[\mathbf{w}_{1} \cdots \mathbf{w}_{n-1}\right]^{H}\left|q_{n}\right|^{2} \mathbf{w}_{n} \\
|\mathbf{p}|^{2} \odot \mathbf{w}_{n}^{H}\left|q_{1}\right|^{2} \mathbf{W}_{n-1} & \left(|\mathbf{p}|^{2} \odot \mathbf{w}_{n}\right)^{H}\left|q_{n}\right|^{2} \mathbf{w}_{n}
\end{array}\right) .
\end{gathered}
$$

From (20), we have

$$
\operatorname{det}\left(\mathbf{I}_{n}+\mathbf{A}_{n}\right) \leq\left(1+\sum_{i}^{n}\left|p_{i}\right|^{2}\left|w_{i n}\right|^{2}\left|q_{n}\right|^{2}\right) \operatorname{det}\left(\mathbf{I}_{n-1}+\mathbf{A}_{n-1}\right) \text {. }
$$

Note that

$$
\operatorname{det}\left(\mathbf{I}_{n}+\mathbf{A}_{n}\right)=\operatorname{det}\left(\mathbf{I}_{n}+\boldsymbol{\Pi}^{H} \mathbf{A}_{n} \boldsymbol{\Pi}\right)
$$

for any permutation matrix $\Pi$. Let $\left|q_{(n)}\right|^{2}=\max \left\{\left|q_{i}\right|^{2}\right\}$; then w.l.o.g., we let $\left|q_{n}\right|^{2}=\left|q_{(n)}\right|^{2}$. Thus

$$
\begin{aligned}
\operatorname{det}\left(\mathbf{I}_{n}+\mathbf{A}_{n}\right) & \leq\left(1+\sum_{i}^{n} p_{i}^{2}\left|w_{i n}\right|^{2} q_{n}^{2}\right) \operatorname{det}\left(\mathbf{I}_{n-1}+\mathbf{A}_{n-1}\right) \\
& \leq\left(1+\left|p_{(n)}\right|^{2}\left|q_{(n)}\right|^{2}\right) \operatorname{det}\left(\mathbf{I}_{n-1}+\mathbf{A}_{n-1}\right)
\end{aligned}
$$

where $\left|p_{(n)}\right|^{2}=\max \left\{\left|p_{i}\right|^{2}\right\}$.

Since $\mathbf{W}_{n-1}^{*}=\boldsymbol{\Pi}_{n-1}^{*}$, by the Hardmard inequality, we have

$$
\left.\max _{\mathbf{W}} \operatorname{det}\left(\mathbf{I}_{n-1}+\mathbf{A}_{n-1}(\mathbf{W})\right)=\prod_{i}^{n-1}\left(1+\left|p_{(i)}\right|^{2}\left|q_{(i)}\right|^{2}\right)\right),
$$

where both $p_{(i)}^{2}$ and $q_{(i)}^{2}$ are sorted in ascending order. Thus

$$
\left.\operatorname{det}\left(\mathbf{I}_{n}+\mathbf{A}_{n}\right) \leq\left(1+\left|p_{(n)}\right|^{2}\left|q_{(n)}\right|^{2}\right) \prod_{i}^{n-1}\left(1+\left|p_{(i)}\right|^{2}\left|q_{(i)}\right|^{2}\right)\right)
$$


with equality if and only if $\mathbf{w}_{n}=[0, \cdots, 0,1,0, \cdots, 0]^{T}$ has entry 1 at the $(n)$ th position. This implies that

$$
\mathbf{W}_{n}=\left[\begin{array}{ll}
\mathbf{W}_{n-1}^{\prime} & \mathbf{w}_{n}
\end{array}\right]
$$

where $\mathbf{W}_{n-1}^{\prime}$ is $\mathbf{W}_{n-1}$ with an additional row of zeros inserted at the $(n)$ th row. Hence, $\mathbf{W}_{n}$ is a permutation matrix. Therefore $\left|\mathbf{I}_{n}+\mathbf{A}_{n}\right|$, i.e., the objective in (19) is maximized by $\mathbf{W}_{n}^{*}=\mathbf{\Pi}_{\mathbf{n}}^{*}$.

Replacing $\Pi$ by $\mathbf{W}$ in (11) and (16), we have the achievable rate $R(\mathbf{W})$ under $\mathbf{W}$ written in the form of (19) for the cases without and with the direct path. Combining Proposition 1 and the results in Section III, the following result immediately follows:

Corollary 1: The optimal $\mathbf{W}^{*}$ in (7) for relaying with or without direct path considered in Section III is $\mathbf{W}^{*}=\mathbf{\Pi}^{*}$.

Corollary 1 shows that, under the assumption of equal power amplification, the optimal linear processing is channel pairing with the optimal pairing strategy based on (12) or (18), depending on whether the direct path is available or not.

\section{Simulation Results}

In this section, we compare the performance of the optimal channel pairing scheme with other non optimal schemes through Monte-Carlo simulations. We consider the scenario of relaying with direct path. We use a $5 \mathrm{MHz}$ OFDM system with $N=128$ subchannels as an example of multichannel system. The achievable rate is averaged over randomly generated multi-tap frequency selective channels . A source-destination pair is placed at a distance $d_{s d}$ apart, and the distances between source and relay, and relay and destination are set at $d_{s r}$ and $d_{r d}$, respectively. The pathloss exponent of 2 is assumed. We assume $P_{s}=P_{r}$, and $P_{s}$ is equally allocated across subchannels, i.e., $d_{s i}=\sqrt{\frac{P_{s}}{N}}$. We denote $\mathrm{SNR} \triangleq \frac{P_{s} d_{s d}^{-2}}{N \sigma_{d}^{2}}$ as the average per subchannel received SNR over the direct path.

We first consider $d_{s d}=20 \mathrm{~m}, d_{s r}=6 \mathrm{~m}$, and $d_{r d}=16 \mathrm{~m}$. Fig. 1 depicts the performance of the average rate per subchannel vs. SNR, averaged over random channel realizations under the following four schemes: 1) optimal channel pairing (CP) scheme $\Pi^{*}$; 2) no channel pairing used, i.e., $\mathbf{W}=\mathbf{I}$; 3) a random $\mathbf{W}$ used; 4) using $\Pi^{*}$ that is obtained assuming no direct path (i.e., (12)). The reason we consider the fourth scheme is that, in some cases, it may be easier for the relay to compute the optimal pairing only based on the SNRs obtained on the two relay paths, although the receiver may use signals from the direct path for combining to improve performance. We see that the optimal channel pairing scheme outperforms all other schemes. When compared with the no pairing scheme, it provides about $1 \mathrm{~dB}$ gain. We expect to see a substantial gap between the optimal channel pairing and other schemes in the highly asymmetric and frequency-selective channels.

Next, we investigate into when channel pairing is the most beneficial. In Fig.2, we show the effect of the relative SNR strengths, between relay and direct paths, on the achievable rate under different linear processing schemes. With fixed

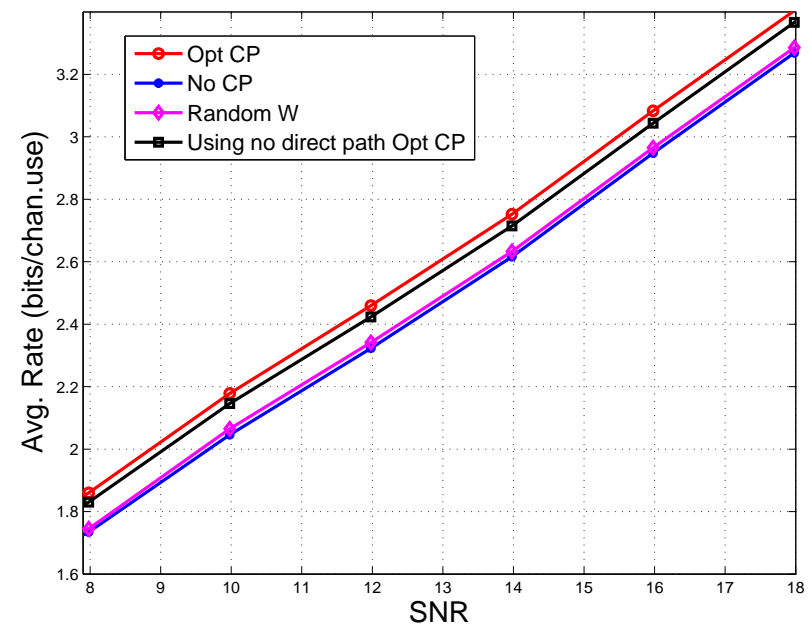

Fig. 1. Relaying with direct path: Rate vs. SNR

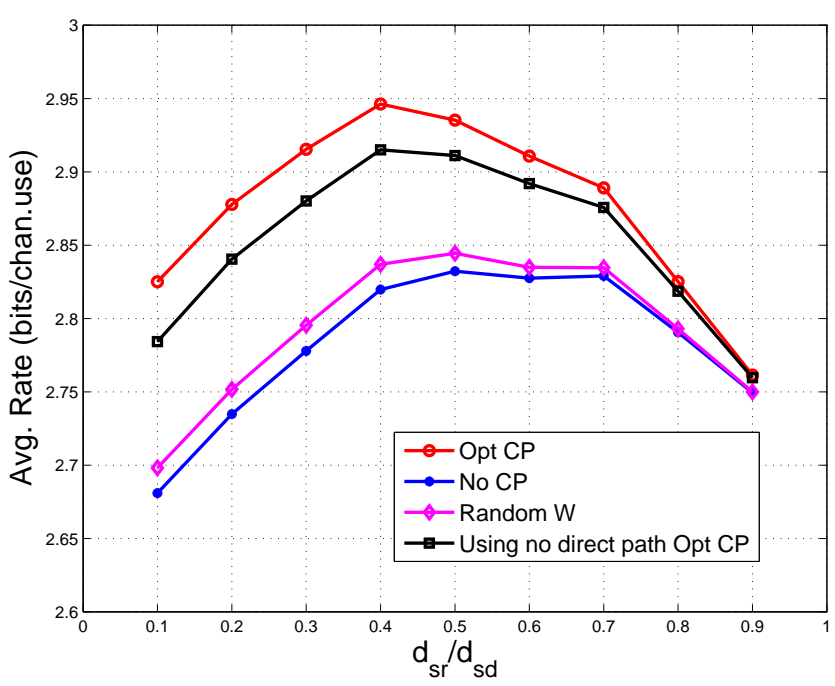

Fig. 2. Relaying with direct path: Rate vs. relative distance $\frac{d_{s r}}{d_{s d}}$

$d_{s d}=20 \mathrm{~m}$, we vary the relay position between source and destination. The average rate vs. the relative distance $d_{s r} / d_{r d}$ for $\mathrm{SNR}=14 \mathrm{~dB}$ is plotted in Fig.2. This figure shows that the performance of all schemes coincide, when relay is very close to destination, i.e., $\mathrm{SNR}_{r d}$ is very high. In this case, channel pairing provides little benefit. On the other hand, when the relay moves closer to source, the gain of using the optimal channel pairing over other schemes become more substantial.

\section{CONCLUSION}

In this paper, AF relaying with linear processing capability at the relay for a multichannel system is considered. We have proposed a method to analyze how to select the linear processing matrix to maximize the end-to-end achievable rate, where equal power amplification over channels at the relay is assumed. Within the class of permutation matrices for channel pairing, this approach allows us to obtain the corresponding optimal permutation for channel pairing both for relaying 
with and without direct path. We have further demonstrated the optimality of the obtained channel pairing scheme in the class of unitary linear processing matrices for achievable rate maximization. Simulation results also demonstrate the gain which can be achieved through optimal channel pairing as compared to the non-optimal linear processing and non-pairing cases.

\section{REFERENCES}

[1] A. Hottinen and T. Heikkinen, "Subchannel assignment in OFDM relay nodes," in Proc. of Annual Conf. on Information Sciences and Systems (CISS), Princeton, NJ, Mar. 2006.

[2] M. Herdin, "A chunk based OFDM amplify-and-forward relaying scheme for $4 \mathrm{G}$ mobile radio systems," in Proc. IEEE Int. Conf. Communications (ICC), June 2006.

[3] A. Hottinen and T. Heikkinen, "Optimal subchannel assignment in a two-hop OFDM relay," in Proc. IEEE Workshop on Signal Processing advances in Wireless Commun.(SPAWC), June 2007.

[4] I. Hammerstrom and A. Wittneben, "Power allocation schemes for amplify-and-forward MIMO-OFDM relay links," IEEE Trans. Wireless Commun., vol. 6, no. 8, pp. 2798-2802, Aug. 2007.

[5] Y. Wang, P. Wang, F. Liu, X. Wang, and X. Yin, "Multiuser subchannel matching algorithms for two-hop OFDM relay networks," in MICNET '09: Proc. of the 1st ACM workshop on Mob. internet through cellular networks. New York, NY, USA: ACM, 2009, pp. 37-42.

[6] W. Wang, S. Yan, and S. Yang, "Optimally joint subcarrier matching and power allocation in OFDM multihop system," EURASIP J. Appl. Signal Process. (USA), Mar. 2008.

[7] W. Wang and R. Wu, "Capacity maximization for OFDM two-hop relay system with separate power constraints," IEEE Trans. Veh. Technol., vol. 58, no. 9, pp. 4943-4954, Nov. 2009.

[8] M. Hajiaghayi, M. Dong, and B. Liang, "Optimal channel pairing and power allocation for multi-channel multi-hop relay networks," in Proc. Int. Conf. on Quality of Service in Heterogeneous Wired/Wireless Networks (QSHINE), Nov. 2010.

[9] W. Dang, M. Tao, H. Mu, and J. Huang, "Subcarrier-pair based resource allocation for cooperative multi-relay OFDM systems," IEEE Trans. Wireless Commun., vol. 9, no. 5, pp. 1640 -1649, May 2010.

[10] M. Hajiaghayi, M. Dong, and B. Liang, "Optimal channel assignment and power allocation for dual-hop multi-channel multi-user relaying," in Proc. IEEE Annual Joint Conf. of the IEEE Computer and Commun. Societies (INFOCOM), 2011.

[11] I. E. Telatar, "Capacity of multi-antenna gaussian channels," EUROPEAN TRANSACTIONS ON TELECOMMUNICATIONS, vol. 10, pp. 585-595, 1999.

[12] Z. Shen, X. Wang, and H. Zhang, "Power allocation and subcarrier pairing for OFDM-based AF cooperative diversity systems," in Proc. IEEE Vehicular Technology Conf. (VTC), Apr. 2009.

[13] T. H. Cormen, C. E. Leiserson, R. L. Rivest, and C. Stein, Introduction to Algorithms, 2nd ed. The MIT Press, September 2001. [Online]. Available: http://www.amazon.com/Introduction-AlgorithmsSecond-Thomas-Cormen/dp/0262531968

[14] F. Holland, "Another proof of Hadamard's determinantal inequality," Bulletin of the Irish Mathematical Society, no. 59, pp. 61-64, 2007. 